\title{
A new solution of the star-triangle relation
}

\author{
Andrew P. Kels \\ Department of Theoretical Physics, Research School of Physics and Engineering, \\ Australian National University, Canberra, ACT 0200, Australia.
}

\begin{abstract}
We obtain a new solution to the star-triangle relation for an Ising-type model with two kinds of spin variables at each lattice site, taking continuous real values and arbitrary integer values, respectively. The Boltzmann weights are manifestly real and positive. They are expressed through the Euler gamma function and depend on sums and differences of spins at the ends of the edge.
\end{abstract}


The star-triangle relation is a distinguished form of the Yang-Baxter equation for Ising-type models on two-dimensional lattices. In these models the fluctuating variables or "spins" are assigned to lattice sites, while two spins interact only if they are connected by an edge of the lattice. Remarkably, many physically interesting models in this class can be solved exactly, for instance, the 2-d Ising [1] and the chiral Potts [2,3] models (see [4,5] for a review of other known cases). The star-triangle relation plays the role of the integrability condition for these models.

Recently, Bazhanov and Sergeev found a very important "master" solution [6] of the startriangle relation, which contains all previously known solutions of this relation as particular cases, and provides many new interesting examples. The above master solution is expressed in terms of the elliptic gamma function. It contains two arbitrary free parameters $\mathrm{p}$ and $\mathrm{q}$, which play the role of elliptic nomes. The spin variables take continuous real values on the circle. Various interesting cases arise when the parameters $\mathrm{p}$ and $\mathrm{q}$ approach some special values. These limits are rather singular and their consideration requires a detailed analysis on a case by case basis (for a comprehensive account see the forthcoming paper [5]). In the present paper we consider one particular remarkable limit of the master solution, which leads to a model with two types of spin variables at each lattice site. These variables take continuous real values and discrete integer values, respectively. Mathematically the master solution of [6] is related to the elliptic beta integral [7]. Here we consider first a degeneration [8] of this solution related to the "hyperbolic beta integral" [9, 10]. Then we consider the so-called "strong coupling regime", where new integer-valued spin variables dynamically arise at each lattice site. Our calculations generalise the results of [11].

Consider the square lattice of $N$ sites and assign spin variables $x_{j}, j=1,2, \ldots, N$, taking some continuous set of values, to all lattice sites. Two spins interact only if they are connected by an edge. Let $\mathcal{W}_{\alpha}(x, y), \overline{\mathcal{W}}_{\alpha}(x, y)$ denote Boltzmann weights for horizontal and vertical edges, where $x$ and $y$ are spins at the end of the edge, as shown in Figure 1. We assume that the edge weights

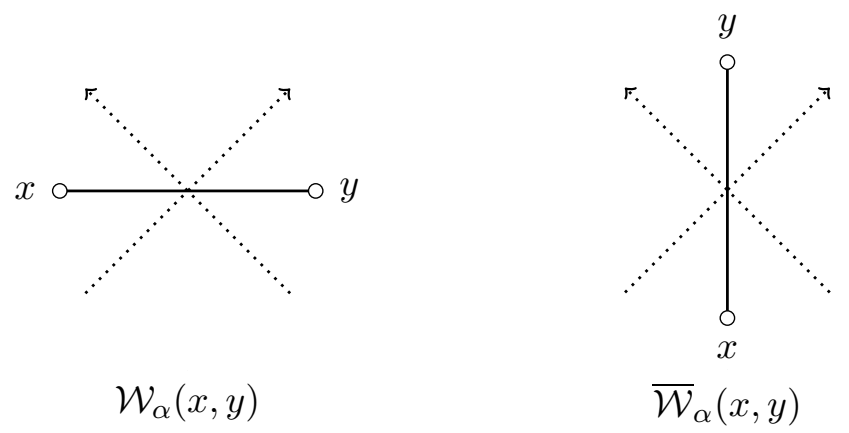

Figure 1: Edges of the first (left) and second types and their Boltzmann weights.

depend on the (additive) spectral variable $\alpha$ and are related to each other by the crossing symmetry $\overline{\mathcal{W}}_{\alpha}(x, y)=\mathcal{W}_{\eta-\alpha}(x, y)$, where $\eta$ is the model dependent crossing parameter. Moreover we assume that the weights are reflection symmetric $\mathcal{W}_{\alpha}(x, y)=\mathcal{W}_{\alpha}(y, x)$. It is also convenient to introduce single-spin weights $\mathcal{S}\left(x_{j}\right)$, which are independent of the spectral variable $\alpha$. The partition function is defined as

$$
\mathcal{Z}=\int \cdots \int \prod_{(i j)} \mathcal{W}_{\alpha}\left(x_{i}, x_{j}\right) \prod_{(k l)} \mathcal{W}_{\eta-\alpha}\left(x_{k}, x_{l}\right) \prod_{m} \mathcal{S}\left(x_{m}\right) d x_{m}
$$

where the first product is taken over all horizontal edges $(i j)$, the second over all vertical edges $(\mathrm{kl})$ and the third over all internal sites of the lattice. We will implicitly assume fixed boundary conditions. Obviously the single spin weights $\mathcal{S}\left(x_{j}\right)$ can be included into the definition of the edge 
weights, but we prefer not to do so. The model is integrable if the weights satisfy the star-triangle equation of the form

$$
\begin{aligned}
\int d x_{0} \mathcal{S}\left(x_{0}\right) \mathcal{W}_{\eta-\alpha_{1}}\left(x_{1}, x_{0}\right) \mathcal{W}_{\eta-\alpha_{2}}\left(x_{2}, x_{0}\right) \mathcal{W}_{\eta-\alpha_{3}}\left(x_{3}, x_{0}\right) \\
=\mathcal{R}\left(\alpha_{1}, \alpha_{2}, \alpha_{3}\right) \mathcal{W}_{\alpha_{1}}\left(x_{2}, x_{3}\right) \mathcal{W}_{\alpha_{2}}\left(x_{1}, x_{3}\right) \mathcal{W}_{\alpha_{3}}\left(x_{2}, x_{1}\right)
\end{aligned}
$$

where the spectral parameters $\alpha_{1}, \alpha_{2}, \alpha_{3}$ satisfy the relation $\alpha_{1}+\alpha_{2}+\alpha_{3}=\eta$ and the factor $\mathcal{R}\left(\alpha_{1}, \alpha_{2}, \alpha_{3}\right)$ is independent of the spins $x_{1}, x_{2}, x_{3}$.

Let us now define the Bazhanov-Sergeev master solution [6]. Introduce the elliptic nomes p, q, and the crossing parameter $\eta$

$$
\mathrm{q}=\mathrm{e}^{\mathrm{i} \pi \tau}, \quad \mathrm{p}=\mathrm{e}^{\mathrm{i} \pi \sigma}, \quad|\mathrm{p}|,|\mathrm{q}|<1, \quad \mathrm{e}^{-2 \eta}=\mathrm{pq}, \quad \eta=-\frac{\mathrm{i} \pi}{2}(\tau+\sigma) .
$$

The elliptic gamma function [12,13] is defined as

$$
\Phi(z)=\prod_{j, k=0}^{\infty} \frac{1-\mathrm{e}^{2 \mathrm{i} z} \mathrm{q}^{2 j+1} \mathrm{p}^{2 k+1}}{1-\mathrm{e}^{-2 \mathrm{i} z} \mathrm{q}^{2 j+1} \mathrm{p}^{2 k+1}}=\exp \left\{\sum_{n \neq 0} \frac{\mathrm{e}^{-2 \mathrm{inx}}}{n\left(\mathrm{p}^{n}-\mathrm{p}^{-n}\right)\left(\mathrm{q}^{n}-\mathrm{q}^{-n}\right)}\right\}
$$

where the exponential form is valid in the strip $|\operatorname{Im} z|<\operatorname{Re} \eta$. The Boltzmann weights are defined by

$$
\mathcal{S}(x)=\frac{\mathrm{e}^{\eta / 2}}{2 \pi} \vartheta_{1}(2 x \mid \mathrm{p}) \vartheta_{1}(2 x \mid \mathrm{q}), \quad \mathcal{W}_{\alpha}(x, y)=\kappa(\alpha)^{-1} \frac{\Phi(x+y+\mathrm{i} \alpha) \Phi(x-y+\mathrm{i} \alpha)}{\Phi(x+y-\mathrm{i} \alpha) \Phi(x-y-\mathrm{i} \alpha)}
$$

where $\vartheta_{1}(z \mid \mathrm{q})$ is the standard Jacobi theta function [14]. Note, that the weights are periodic

$$
\mathcal{W}_{\alpha}(x+\pi, y)=\mathcal{W}_{\alpha}(x, y+\pi)=\mathcal{W}_{\alpha}(x, y), \quad \mathcal{S}(x+\pi)=\mathcal{S}(x)
$$

Correspondingly, the spin variables in (1) take values in the interval $0 \leq x_{m}<\pi$. The normalisation factor $\kappa(\alpha)$ in (5) reads

$$
\log \kappa(\alpha)=\sum_{n \neq 0} \frac{\mathrm{e}^{4 \alpha n}}{n\left(\mathrm{p}^{n}-\mathrm{p}^{-n}\right)\left(\mathrm{q}^{n}-\mathrm{q}^{-n}\right)\left(\mathrm{p}^{n} \mathrm{q}^{n}+\mathrm{p}^{-n} \mathrm{q}^{-n}\right)} .
$$

The above Boltzmann weights satisfy the star-triangle relation (2) where the integration is taken over the segment $0 \leq x_{0}<\pi$. Below we assume that the spectral parameter $\alpha$ lies in the domain $0<\alpha<\eta$. In this case the weights (15) are real and positive.

With the normalisation (7) the factor $\mathcal{R}$ in (2) is equal to one. For the same normalisation, the weights (5) also satisfy the following inversion relations

$$
\begin{aligned}
\mathcal{W}_{\alpha}(x, y) \mathcal{W}_{-\alpha}(x, y) & =1 \\
\int_{0}^{\pi} d z \mathcal{S}(z) \mathcal{W}_{\eta-\alpha}(x, z) \mathcal{W}_{\eta+\alpha}(z, y) & =\frac{1}{2 \mathcal{S}(x)}(\delta(x+y)+\delta(x-y)) .
\end{aligned}
$$

These relations allow one to show [6] that in the thermodynamic limit, when the number of lattice sites goes to infinity $N \rightarrow \infty$, the bulk free energy of the system vanishes,

$$
\lim _{N \rightarrow \infty} N^{-1} \log \mathcal{Z}=0 .
$$


As a mathematical identity the star-triangle relation (2) is equivalent to the elliptic beta integral [7, which plays a fundamental role in the theory of elliptic hypergeometric functions. Its significance for solvable models of statistical mechanics was discovered in [6].

Now consider the hyperbolic limit [8] of (55). Introduce the modular parameter $b$ to take values in one of the following two regimes

$$
\text { (i) } \mathrm{b}>0, \quad(i i)|\mathrm{b}|=1, \quad \operatorname{Im}\left(\mathrm{b}^{2}\right)>0 .
$$

The non-compact quantum dilogarithm is defined as the integral

$$
\varphi(z)=\exp \left\{\frac{1}{4} \int_{\mathbb{R}+\mathrm{i} 0} \frac{\mathrm{e}^{-2 \mathrm{i} y z} d y}{y \sinh (y \mathrm{~b}) \sinh (y / \mathrm{b})}\right\}, \quad|\operatorname{Im}(z)|<\operatorname{Re}(\eta),
$$

where the singularity at the origin is taken below the contour. Substituting

$$
\mathrm{p}=\mathrm{e}^{-b \epsilon}, \quad \mathrm{q}=\mathrm{e}^{-b^{-1} \epsilon}, \quad x_{i}=x_{i} \epsilon, \quad \alpha_{i}=\alpha_{i} \epsilon, \quad i=1,2,3,
$$

into (5) and taking the limit $\epsilon \rightarrow 0$, one obtains $[8$,

$$
\mathcal{S}(x)=2 \sinh \left(2 \pi x_{0} \mathrm{~b}\right) \sinh \left(2 \pi x_{0} / \mathrm{b}\right), \quad \mathcal{W}_{\alpha}(x, y)=\kappa(\alpha)^{-1} \mathrm{e}^{4 \pi \alpha x} \frac{\varphi(x+y+\mathrm{i} \alpha)}{\varphi(x+y-\mathrm{i} \alpha)} \frac{\varphi(x-y+\mathrm{i} \alpha)}{\varphi(x-y-\mathrm{i} \alpha)} .
$$

These weights satisfy the star-triangle relation (2), where the integration taken over the whole real line and the crossing parameter is given by $\eta=\left(\mathrm{b}+\mathrm{b}^{-1}\right) / 2$. The normalisation factor $\kappa(\alpha)$ in (13) reads

$$
\log \kappa(\alpha)=\frac{1}{8} \int_{\mathbb{R}+\mathrm{i} 0} \frac{\mathrm{e}^{4 y z} d y}{y \sinh (y \mathrm{~b}) \sinh (y / \mathrm{b}) \cosh (2 y \eta)}+\pi \mathrm{i} \alpha^{2}-\frac{\pi \mathrm{i}}{3} \eta^{2}+\frac{\pi \mathrm{i}}{24} .
$$

The weights (13) define a physical model of statistical mechanics, rather similar to the FaddeevVolkov model [15, 16]. The spins now take arbitrary values on the real line $x_{j} \in \mathbb{R}$. The above limiting procedure does not affect the bulk free energy of the system, so that the relation (9) continues to hold for the new model.

Let us now consider a further specialisation of the above generalised Faddeev-Volkov model (13), when the parameter $\mathrm{b} \rightarrow \mathrm{i}$, or equivalently, $\eta \rightarrow 0$. In this limit the weights $\mathcal{W}_{\alpha}(x, y)$ develop a series of delta function like peaks for integer values of the sum $x+y \in \mathbb{Z}$ or differences $x-y \in \mathbb{Z}$ of the arguments $x, y$. This limit was previously investigated for the Faddeev-Volkov model [11] and a similar phenomenon was observed. A correct limiting procedure capturing a fine structure of these peaks requires a redefinition of the spin variables. The asymptotics of the non-compact quantum dilogarithm $\varphi(z)$ and the function $\kappa(\alpha)$ read

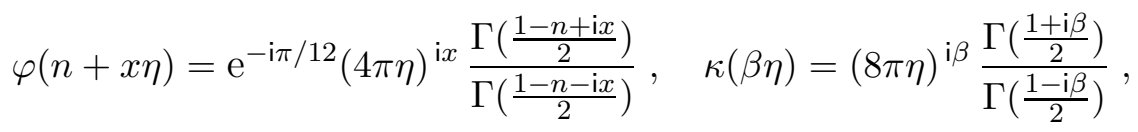

where $\eta \rightarrow 0, \quad n \in \mathbb{Z}, \quad x \ll \eta^{-1}$ and $0<\beta<1$. Using this in (13) one obtains the following $\eta \rightarrow 0$ asymptotics 1

$$
\begin{aligned}
\mathcal{S}(n+x \eta) & \rightarrow 8 \pi^{2}\left(x^{2}+n^{2}\right) \eta^{2} \\
\mathcal{W}_{\beta \eta}(m+x \eta, n+y \eta) & \rightarrow \frac{2^{-5 \beta}}{(\pi \eta)^{-3 \beta}} \frac{\Gamma\left(\frac{1+\beta}{2}\right)}{\Gamma\left(\frac{1-\beta}{2}\right)} \frac{\Gamma\left(\frac{1-\beta}{2} \pm \frac{\mathrm{i}(x+y)-(m+n)}{2}\right) \Gamma\left(\frac{1-\beta}{2} \pm \frac{\mathrm{i}(x-y)-(m-n)}{2}\right)}{\Gamma\left(\frac{1+\beta}{2} \pm \frac{\mathrm{i}(x+y)+(m+n)}{2}\right) \Gamma\left(\frac{1+\beta}{2} \pm \frac{\mathrm{i}(x-y)+(m-n)}{2}\right)},
\end{aligned}
$$

\footnotetext{
${ }^{1}$ Here we use the compact notation, where the \pm symbol in the argument of the gamma function indicates the product of gamma functions with both signs are taken as e.g. $\Gamma(a \pm b) \equiv \Gamma(a+b) \Gamma(a-b)$
} 
where $m, n \in \mathbb{Z}$ and $x, y \in \mathbb{R}$. Substituting this into (2) and cancelling out singular factors from both sides, one obtains the following star-triangle relation

$$
\begin{array}{r}
\sum_{n_{0} \in \mathbb{Z}} \int_{-\infty}^{\infty} d x_{0} \mathcal{S}\left(x_{0}, n_{0}\right) \mathcal{W}_{1-\beta_{1}}\left(x_{1}, n_{1} \mid x_{0}, n_{0}\right) \mathcal{W}_{1-\beta_{2}}\left(x_{2}, n_{2} \mid x_{0}, n_{0}\right) \mathcal{W}_{1-\beta_{3}}\left(x_{3}, n_{3} \mid x_{0}, n_{0}\right) \\
=\mathcal{W}_{\beta_{1}}\left(x_{2}, n_{2} \mid x_{3}, n_{3}\right) \mathcal{W}_{\beta_{2}}\left(x_{1}, n_{1} \mid x_{3}, n_{3}\right) \mathcal{W}_{\beta_{3}}\left(x_{2}, n_{2} \mid x_{1}, n_{1}\right)
\end{array}
$$

where $\beta_{1}+\beta_{2}+\beta_{3}=1$, and the Boltzmann weights are given by

$\mathcal{S}(x, n)=\frac{1}{2 \pi}\left(x^{2}+n^{2}\right), \quad \mathcal{W}_{\beta}(x, n \mid y, m)=\frac{\Gamma\left(\frac{1+\beta}{2}\right)}{\Gamma\left(\frac{1-\beta}{2}\right)} \frac{\Gamma\left(\frac{1-\beta}{2} \pm \frac{\mathrm{i}(x+y)-(m+n)}{2}\right) \Gamma\left(\frac{1-\beta}{2} \pm \frac{\mathrm{i}(x-y)-(m-n)}{2}\right)}{\Gamma\left(\frac{1+\beta}{2} \pm \frac{\mathrm{i}(x+y)+(m+n)}{2}\right) \Gamma\left(\frac{1+\beta}{2} \pm \frac{\mathrm{i}(x-y)+(m-n)}{2}\right)}$.

These weights give a new solution of the star-triangle relation for an Ising type model, where sites of the lattice are assigned "dual spin" variables $\boldsymbol{x}$

$$
\boldsymbol{x}=(x, n), \quad x \in \mathbb{R}, \quad n \in \mathbb{Z},
$$

taking arbitrary real and integer values, respectively. The Boltzmann weights (18) satisfy the spin reflection symmetry $\boldsymbol{x} \leftrightarrow \boldsymbol{y}$, for two spins $\boldsymbol{x}, \boldsymbol{y}$ connected by an edge as in Figure 11. The bulk free energy (9) of the corresponding 2-d lattice model remains unchanged.

The star-triangle relation (17) is derived in the strong-coupling limit of the generalised FaddeevVolkov model (13), the latter model arising as a hyperbolic degeneration of the master solution (5). From either of the models (5), (13), new solutions of the star-triangle relation can be found as different singular limits of the parameters $\mathrm{p}, \mathrm{q}$, and $\mathrm{b}$ respectively. In each case, the spins and spectral parameters should be redefined, as was done here in (12) and (16), in order to obtain convergent limits.

\section{Acknowledgments}

I thank Vladimir Bazhanov for suggesting the problem and useful advice.

\section{References}

[1] Baxter, R. J. Exactly Solved Models in Statistical Mechanics. Academic, London, 1982.

[2] Au-Yang, H., McCoy, B. M., perk, J. H. H., Tang, S., and Yan, M.-L. Commuting transfer matrices in the chiral Potts models: Solutions of star-triangle equations with genus $>1$. Phys. Lett. A123 (1987) 219-223.

[3] Baxter, R. J., Perk, J. H. H., and Au-Yang, H. New solutions of the star triangle relations for the chiral Potts model. Phys. Lett. A128 (1988) 138-142.

[4] Baxter, R. J. A rapidity-independent parameter in the star-triangle relation. In MathPhys Odyssey, 2001, volume 23 of Prog. Math. Phys., pages 49-63. Birkhäuser Boston, Boston, MA, 2002 .

[5] Bazhanov, V. V., Kels, A. P., and Sergeev, S. M. Quasi-classical expansion of the star-triangle relation and integrable systems on quad-graphs. In preparation (2013). 
[6] Bazhanov, V. V. and Sergeev, S. M. A master solution of the quantum Yang-Baxter equation and classical discrete integrable equations. Adv. Theor. Math. Phys. 16 (2012) 65-95.

[7] Spiridonov, V. P. On the elliptic beta function. Uspekhi Mat. Nauk 56 (2001) 181-182.

[8] Spiridonov, V. P. Elliptic beta integrals and solvable models of statistical mechanics. Contemp. Math. 563 (2012) 181-211.

[9] Rains, E. M. Limits of elliptic hypergeometric integrals. Ramanujan J. 18 (2006) 257-306.

[10] Stokman, J. V. Hyperbolic beta integrals. Adv. in Math. 190 (2004) 119-160.

[11] Bazhanov, V. V., Mangazeev, V. V., and Sergeev, S. M. Exact solution of the Faddeev-Volkov model. Phys. Lett. A372 (2008) 1547-1550.

[12] Ruijsenaars, S. N. M. First order analytic difference equations and integrable quantum systems. J. Math. Phys. 38 (1997) 1069-1146.

[13] Felder, G. and Varchenko, A. The elliptic gamma function and $\operatorname{SL}(3, \mathbf{Z}) \ltimes \mathbf{Z}^{3}$. Adv. Math. 156 (2000) 44-76.

[14] Whittaker, E. and Watson, G. A course of modern analysis. Merchant Books, 1915.

[15] Volkov, A. Y. and Faddeev, L. D. Yang-Baxterization of the quantum dilogarithm. Zapiski Nauchnykh Seminarov POMI 224 (1995) 146-154. English translation: J. Math. Sci. 88 (1998) 202-207.

[16] Bazhanov, V. V., Mangazeev, V. V., and Sergeev, S. M. Faddeev-Volkov solution of the Yang-Baxter equation and discrete conformal symmetry. Nucl. Phys. B784 (2007) 234-258. 